\title{
Fuzzy Bitopological Ideals Spaces
}

\author{
M. E. Abd El-Monsef ${ }^{1}$; A.Kozae ${ }^{2}$; A. A. Salama ${ }^{3}$ and H.M.Elagmy ${ }^{4}$ \\ ${ }^{3}$ Dept. of Math and Computer Sci., Faculty of Science, Port Said Univ., Egypt \\ ${ }^{1,2.4}$ Dept. Of Math., Faculty of Science, Tanta Univ., Egypt
}

\begin{abstract}
In this paper we introduce the notion of fuzzy bitopological ideals. The concept of fuzzy pairwise local function is also introduced here by utilizing the q-neighborhood structure for a fuzzy topological space .These concepts are discussed fuzzy bitopologies and several relations between different fuzzy bitopological ideals.
\end{abstract}

Keywords: Fuzzy bitopological space; fuzzy ideals; fuzzy pairwise local function

\section{Introduction}

The concept of fuzzy sets and fuzzy set operations was first introduced by Zadeh [6]. Subsequently, Chang defined the notion of fuzzy topology [2]. Since then various aspects of general topology were investigated and. carried out in fuzzy since by several authors of this field. The notions of fuzzy ideal and fuzzy local function introduced and studied in [5]. Nouh [ 4 ] initiated the study of fuzzy bitopological spaces . A fuzzy set equipped with two fuzzy topologies is called a fuzzy bitopological space. Concepts of fuzzy ideals and fuzzy local function were introduced by Sarkar [5].The purpose of this paper is to suggest the fuzzy ideals in fuzzy bitopological space. The concept of fuzzy pairwise local function is also introduced here by utilizing the q-neighborhood structure [3].

\section{Preliminaries}

Throughout this paper, by $\left(\mathrm{X}, \tau_{1}, \tau_{2}\right)$ we mean a fuzzy bitopological space (fbts in short) in Nouh's [ 4 ] sense. A fuzzy point in $\mathrm{X}$ with support $\mathrm{x} \in \mathrm{X}$ and value $\varepsilon(0<\varepsilon \leq 1)$ is denoted by $\mathrm{x}_{\mathcal{E}}$ [1]. A fuzzy point $\mathrm{x}_{\mathcal{E}}$ is said to be contained in a fuzzy set $\mu$ in $\mathrm{I}^{\mathrm{X}}$ iff $\varepsilon \leq \mu$ and this will be denoted by $\mathrm{x}_{\varepsilon} \in \mu$ [3] . For a fuzzy set $\mu$ in a fbts $\left(\mathrm{X}, \tau_{1}, \tau_{2}\right), \tau_{i}-\operatorname{cl}(\mu), \tau_{i}-\operatorname{Int}(\mu), i \in\{1,2\}$, and $\mu^{\mathrm{c}}$ will respectively denote closure, interior and complement of $\mu$. The constant fuzzy sets taking values 0 and 1 on $X$ are denoted by $o_{x}, 1_{x}$ respectively. A fuzzy set $\mu$ in fts is said to be quasi-coincident [3] with a fuzzy set $\eta$, denoted by $\mu$ q $\eta$, if there exists $x \in X$ such that $\mu(x)+\eta(x)>1$. A fuzzy set $\nu$ in a fts $(X, \tau)$ is called a q-nbd [1,3] of a fuzzy point $x_{\varepsilon}$ iff there exists a fuzzy open set $\mu$ such that $x_{\varepsilon} q \mu \subseteq V$ we will denoted the set of all q-nbd of $x_{\varepsilon}$ in $(X, \tau)$ by $N(X, \tau)$. A nonempty collection of fuzzy sets $L$ of a set $X$ is called fuzzy ideal [5] on $X$ iff i) $\mu \in$ $\mathrm{L}$ and $\eta \subseteq \mu \Rightarrow \mu \in \mathrm{L}$ (heredity), (ii) $\mu \in \mathrm{L}$ and $\eta \in \mathrm{L} \Rightarrow \mu \vee \eta \in \mathrm{L}$ (finite additivity). The fuzzy local function [5] $\mu^{*}(L, \tau)$ of a fuzzy set $\mu$ is the union of all fuzzy points $x_{\varepsilon}$ such that if $\nu \in N\left(x_{\varepsilon}\right)$ and $\rho \in L$ then there is at least one $r \in X$ for which $V(r)+\mu(r)-1>\rho(r)$. For a fts $(X, \tau)$ with fuzzy ideal $L$ $\mathrm{cl}^{*}(\mu)=\mu \vee \mu^{*}[5]$ for any fuzzy set $\mu$ of $X$ and $\tau^{*}(\mathrm{~L})$ be the fuzzy topology generated by cl ${ }^{*}$ [5].

\section{Fuzzy Pair wise Local Functions.}

Definition 2.1. A fuzzy set $\mu$ in a fbts $\left(X, \tau_{1}, \tau_{2}\right)$ is called Pairwise Quasi-coincident with a fuzzy set $\eta$. denoted by $P(\mu q \eta)$, if there exists $x \in X$ such that $\mu(x)+\eta(x)>1$.Obviously, for any two fuzzy sets $\mu$ and $\eta, P(\mu q \eta)$ will imply $P(\eta q \mu)$.

Definition 2.2. A fuzzy set $\mu$ in a fbts $\left(\mathrm{X}, \tau_{1}, \tau_{2}\right)$ is called pairwise quasi-neighborhood of point $x_{\varepsilon}$ if and only if there exists a fuzzy $\tau_{i}$-open, $i \in\{1,2\}$ set $\rho$ such that $x_{\varepsilon} q \rho \subseteq \mu$ We will denote the set of all pairwise q-nbd of $x_{\varepsilon}$ in $\left(\mathrm{X}, \tau_{1}, \tau_{2}\right)$ by $P N\left(x_{\varepsilon}, \tau_{i}\right), i \in\{1,2\}$. 
Definition 2.3. Let $\left(\mathrm{X}, \tau_{1}, \tau_{2}\right)$ be a fbts with fuzzy ideal $\mathrm{L}$ on $\mathrm{X}$, and $\mu \in I^{X}$. Then the fuzzy pairwise local function $P \mu^{*}(\mathrm{~L}, \tau), i \in\{1,2\}$ of $\mu$ is the union of all fuzzy points $\mathrm{x}_{\varepsilon}$ such that for $\rho \in \mathrm{PN}\left(\mathrm{x}_{\varepsilon}, \tau\right)$ and $\ell \in \mathrm{L}$ then there is at least one $\mathrm{r} \in \mathrm{X}$ for which $\rho(r)+\mu(r)-1>\ell(r)$, where $\mathrm{PN}\left(\mathrm{x}_{\varepsilon}, \tau\right)$ is the set of all qnbd of $x_{\varepsilon}$. Therefore, any $x_{\varepsilon} \notin P \mu^{*}(\mathrm{~L}, \tau i), i \in\{1,2\}$ ( for any $x_{\varepsilon} \notin \mu$ ( any fuzzy set ) implies hereafter, $x_{\varepsilon}$ is not contained in the fuzzy set $\mu$, i.e. $\varepsilon>\mu(x)$ implies there is at least one $\rho \in \mathrm{PN}\left(\mathrm{x}_{\varepsilon}\right)$ such that for every $\mathrm{r} \in \mathrm{X}, \rho(\mathrm{r})+\mu(\mathrm{r})-1 \leq \ell(\mathrm{r})$ for some $\ell \in \mathrm{L}$.

We will occasionally write $P \mu^{*}$ or $P \mu^{*}(\mathrm{~L})$ for $P \mu^{*}\left(\mathrm{~L}, \tau_{i}\right)$.We define $P$ *-fuzzy closure operator, denoted by pcl for fuzzy topology $\tau^{*}{ }_{i}$ (L) finer than $\tau_{i}$ as follows: $\operatorname{pcl}^{*}(\mu)=\mu \vee P \mu{ }^{*}$ for every fuzzy set $\mu$ on $\mathrm{X}$. When there is no ambiguity, we will simply write for $P \mu^{*}$ and $\tau_{i}{ }^{*}$ for $P \mu^{*}(\mathrm{~L}, \tau i)$ and $\tau_{i}{ }^{*}(\mathrm{~L})$, respectively.

Definition 2.4. Let $\left(\mathrm{X}, \tau_{1}, \tau_{2}\right)$ be a fbts with fuzzy ideal $\mathrm{L}$ on $\mathrm{X}$, a fuzzy pairwise local function $P \mu^{*}$ (L, $\left.\tau_{1} \vee \tau_{2}\right), i \in\{1,2\}$ of $\mu \in I^{X}$ is the union of all fuzzy points $\mathrm{x}_{\varepsilon}$ such that for $\rho \in \mathrm{PN}\left(\mathrm{x}_{\varepsilon}, \tau i\right)$ and $\ell \in \mathrm{L}$ then there is at least one $\mathrm{r} \in \mathrm{X}$ for which $\rho(r)+\mu(r)-1>\ell(r)$, where PN $\left(\mathrm{x}_{\varepsilon}, \tau\right)$ is the set of all q-nbd of $x_{\varepsilon}$ in $\tau_{1} \vee \tau_{2}$ (where $\tau_{1} \vee \tau_{2}$ is fuzzy topology generated by $\tau_{1}, \tau_{2}$.

Example 2.1. One may easily verify that

i- $\quad \mathrm{L}=\left\{0_{\mathrm{x}}\right\}$, then $P \mu^{*}\left(\mathrm{~L}, \tau_{i}\right)=\tau_{i}-c l(\mu)$, for any $\mu \in I^{X}, i \in\{1,2\}$.

ii- $L=I^{X}$, then $P \mu^{*}\left(\mathrm{~L}, \tau_{i}\right)=0_{X}$, for any $\mu \in I^{X}, i \in\{1,2\}$.

Note 2.1. In a fbts $\left(\mathrm{X}, \tau_{1}, \tau_{2}\right)$ with fuzzy ideal $\mathrm{L}$ on $\mathrm{X}$, we will denote by $\sigma-\operatorname{cl}(\mu)($ resp. $\sigma-\operatorname{int}(\mu))$ the closure (resp. interior) of a fuzzy subset $\mu \in I^{X}$ with respect to the fuzzy topology $\sigma=\tau_{1} \vee \tau_{2}$.

The following theorem gives some general properties of fuzzy pairwise-local function.

Theorem 2.1. Let $\left(\mathrm{X}, \tau_{1}, \tau_{2}\right)$ be a fbts with fuzzy ideal $\mathrm{L}$ on $\mathrm{X}, \mu, \eta \in I^{X}$ and $\sigma=\tau_{1} \vee \tau_{2}$. Then we have:

i- $\quad P \mu^{*}(\mathrm{~L}, \sigma) \subseteq P \mu^{*}\left(\mathrm{~L}, \tau_{i}\right) ; i \in\{1,2\}$.

ii- $\quad$ If $\mu \subseteq \eta$ then $P \mu^{*}(\mathrm{~L}, \sigma) \subseteq P \eta^{*}\left(\mathrm{~L}, \tau_{i}\right) ; i \in\{1,2\}$.

iii- $\quad P \mu^{*}(\mathrm{~L}, \sigma) \subseteq \sigma-\operatorname{cl}(\mu) \subseteq \tau_{i-} \operatorname{cl}(\mu)$.

iv- $\quad P \mu^{* *}(\mathrm{~L}, \sigma) \subseteq P \mu^{*}\left(\mathrm{~L}, \tau_{i}\right) ; i \in\{1,2\}$.

\section{Proof}

i- $\quad$ Let $\mathrm{x}_{\varepsilon} \notin P \mu^{*}\left(\mathrm{~L}, \tau_{i}\right)$ i.e. $\varepsilon>P \mu^{*}(x)$ so $\mathrm{x}_{\varepsilon}$ is not contained in $P \mu^{*}$

This implies there is at least one $\rho \in \mathrm{PN}\left(\mathrm{x}_{\varepsilon}\right)$ in $\tau_{i}$ such that for every $\mathrm{r} \in \mathrm{X}$,

$\rho(\mathrm{r})+\mu(\mathrm{r})-1 \leq \ell(\mathrm{r})$ for some $\ell \in \mathrm{L}$. Hence $\rho \in P N\left(x_{\varepsilon}, \sigma\right)$ and so $x_{\varepsilon} \notin P \mu^{*}(\mathrm{~L}, \sigma)$. Therefore $P \mu^{*}(\mathrm{~L}, \sigma) \subseteq P \mu^{*}\left(\mathrm{~L}, \tau_{i}\right) ; i \in\{1,2\}$.

ii- $\quad x_{\varepsilon} \in P \eta^{*}\left(\mathrm{~L}, \tau_{i}\right) ; i \in\{1,2\}$, then $\varepsilon \leq P \eta^{*}(x)$, so $\mathrm{x}_{\varepsilon}$ is contained in $P \eta^{*}$. This implies there is at least one q-nbd $\rho \in P N\left(x_{\varepsilon}, \tau_{i}\right)$ such that every $\mathrm{r} \in \mathrm{X}$,

$\rho(\mathrm{r})+\eta(\mathrm{r})-1>\ell(\mathrm{r}), \ell \in \mathrm{L}$. Hence $\rho \in P N\left(x_{\varepsilon}, \sigma\right)$. Since $\mu \subseteq \eta$, by heredity $\rho(\mathrm{r})+\mu(\mathrm{r})-1>\ell(\mathrm{r}), \ell \in \mathrm{L}$. Therefore $x_{\varepsilon} \notin P \mu^{*}(\mathrm{~L}, \sigma)$

iii- $\quad$, (iv) Obvious. 
Theorem 2.2. Let $\left(X, \tau_{1}, \tau_{2}\right)$ be a fbts with fuzzy ideal $L$ on $X, \mu \in I^{X}$, If $\tau_{1} \subseteq \tau_{2}$, then i- $\quad P \mu^{*}\left(\mathrm{~L}, \tau_{2}\right) \subseteq P \mu^{*}\left(\mathrm{~L}, \tau_{1}\right)$, for every $\mu \in I^{X}, \tau_{1}^{*} \subseteq \tau_{2}^{*}$.

Proof .Since every q-nbd in $\tau_{1}$ of any fuzzy point $x_{\varepsilon}$ is also q-nbd in $\tau_{2}$. Therefore, $P \mu^{*}\left(\mathrm{~L}, \tau_{2}\right) \subseteq$ $P \mu^{*}\left(\mathrm{~L}, \tau_{1}\right)$ as there may be other q-nbd in $\tau_{2}$ of $x_{\varepsilon}$ where is condition for $x_{\varepsilon} \in P \mu^{*}\left(\mathrm{~L}, \tau_{2}\right)$ may not hold true, although $x_{\varepsilon} \in P \mu^{*}\left(\mathrm{~L}, \tau_{1}\right)$.

ii- $\quad$ Cleary, $\tau_{1}^{*} \subseteq \tau_{2}^{*}$ as $P \mu^{*}\left(\mathrm{~L}, \tau_{2}\right) \subseteq P \mu^{*}\left(\mathrm{~L}, \tau_{1}\right)$.

Theorem 2.3 . Let $\left(\mathrm{X}, \tau_{1}, \tau_{2}\right)$ be a fbts and $\mathrm{L}, \mathrm{J}$ be two fuzzy ideals on $\mathrm{X}$,. Then for any fuzzy sets $\mu, \rho \in I^{X}$.

i- $\quad \mu \subseteq \rho \Rightarrow P \mu^{*}\left(\mathrm{~L}, \tau_{i}\right) \subseteq P \rho^{*}\left(\mathrm{~L}, \tau_{i}\right), i \in\{1,2\}$.

ii- $\quad L \subseteq J \Rightarrow P \mu^{*}\left(\mathrm{~J}, \tau_{i}\right) \subseteq P \mu^{*}\left(\mathrm{~L}, \tau_{i}\right), i \in\{1,2\}$,

iii- $P \mu^{*}=\tau_{i}-c l\left(P \mu^{*}\right) \subseteq, \tau_{i}-c l(\mu), i \in\{1,2\}$,

iv- $P \mu^{* *}\left(\mathrm{~L}, \tau_{i}\right) \subseteq P \mu^{*}\left(\mathrm{~L}, \tau_{i}\right), i \in\{1,2\}$,

v- $P(\mu \cup \rho)^{*}\left(\mathrm{~L}, \tau_{i}\right)=P \mu^{*}\left(\mathrm{~L}, \tau_{i}\right) \cup \rho^{*}\left(L, \tau_{i}\right)$.

vi- $\quad \rho \in L \Rightarrow P(\mu \cup \rho)^{*}\left(\mathrm{~L}, \tau_{i}\right)=P \mu^{*}\left(\mathrm{~L}, \tau_{i}\right)$.

Proof.

i- $\quad$ Since $\mu \subseteq \rho$ implies $\mu(x) \leq \rho(x)$ for every $x$ in $X$, therefore by Definition 2.1 $x_{\varepsilon} \in P \mu^{*}\left(\mathrm{~L}, \tau_{i}\right)$ implies $x_{\varepsilon} \in P \rho^{*}\left(\mathrm{~L}, \tau_{i}\right)$, which complete the proof of (i)

ii- $\quad$ Cleary, $L \subseteq J \Rightarrow P \mu^{*}\left(\mathrm{~J}, \tau_{i}\right) \subseteq P \mu^{*}\left(\mathrm{~L}, \tau_{i}\right), i \in\{1,2\}$ as there may be other fuzzy sets which belong to $J$ so that for a fuzzy point $x_{\varepsilon} \in P \mu^{*}\left(\mathrm{~J}, \tau_{i}\right)$ but $x_{\varepsilon}$ may not be contained in $P \mu^{*}\left(\mathrm{~L}, \tau_{i}\right)$.

iii- $\quad$ Since $\left\{0_{x}\right\} \subseteq L$ for any fuzzy ideal $L$ on $X$, Therefore by (ii) and Example $2.1, P \mu^{*}\left(\mathrm{~L}, \tau_{i}\right) \subseteq$ $P \mu^{*}\left(\left\{0_{x}\right\}, \tau_{i}\right)=\tau_{i}-c l(\mu)$ for any fuzzy set $\mu$ of $X$. Suppose, $x_{\varepsilon} \in \tau_{i-} c l\left(\mu^{*}\right)$, so there is at is at least one $\mathrm{r} \in \mathrm{X}$ for which $\mu^{*}(r)+v(r)-1>\ell(r)$, for each q-nbd $v$ of $x_{\varepsilon}$.Hence $\mu^{*}(r) \neq 0_{x}$. Let $S=\mu^{*}(r)$. Cleary $r_{t} \in P \mu^{*}\left(\mathrm{~L}, \tau_{i}\right)$ and $t+v(r)>1$ so that $v$ is also q-nbd of $r_{t}$ in $\tau_{i}$. Now $r_{t} \in p \mu^{*}\left(L, \tau_{i}\right)$, So there is at is at least one $r^{\prime} \in X$ for which $\eta\left(r^{\prime}\right)+\mu\left(r^{\prime}\right)-1>\ell\left(r^{\prime}\right)$, for each qnbd $\eta$ of $r_{t}$ and $\ell \in L$. this is also true for $v$ so there is at is at least one $r^{\prime \prime} \in X$ such that $v\left(r^{\prime \prime}\right)+\mu\left(r^{\prime \prime}\right)-1>\ell\left(r^{\prime \prime}\right)$, for each $\ell \in L$. Since $v$ is an arbitrary q-nbd of $x_{\varepsilon}$ in $\tau_{i}$ therefore $x_{\varepsilon} \in p \mu^{*}\left(L, \tau_{i}\right)$ hence, $P \mu^{*}=\tau_{i}-c l\left(P \mu^{*}\right) \subseteq, \tau_{i}-c l(\mu), i \in\{1,2\}$,

iv- Clear

v- Suppose, $\mathrm{x}_{\varepsilon} \notin, P \mu^{*}\left(\mathrm{~L}, \tau_{i}\right) \cup \rho^{*}\left(L, \tau_{i}\right)$ i-e. $\varepsilon>\left(\mathrm{P} \mu^{*} \vee \mathrm{P} \rho^{*}\right)(\mathrm{x})=\max \left\{P \mu^{*}(\mathrm{x}), \mathrm{P} \rho^{*}(\mathrm{x})\right\}$. So $\mathrm{x}_{\varepsilon}$ is not contained in both $P \mu^{*}$ and $P \rho^{*}$. This implies there is at least one q-nbd $v_{1}$ in $\tau_{i}$, of $\mathrm{x}_{\varepsilon}$ such that for every $\mathrm{r} \in \mathrm{X}, \boldsymbol{V}_{1}(\mathrm{r})+\mu(\mathrm{r})-1 \leq_{e}(\mathrm{r})$, for some $\ell_{1} \in \mathrm{L}$ and similarly, there is at least one qnbd $v_{2}$ of $\mathrm{x}_{\varepsilon}$ in $\tau_{i}$ such that, for every $\mathrm{r} \in \mathrm{X}, \boldsymbol{V}_{2}(\mathrm{r})+\rho(\mathrm{r})-1 \leq \ell_{2}(\mathrm{x})$ for some $\ell_{2} \in \mathrm{L}$. Let $v=$ $v_{1} \wedge v_{2}$, so $v$ is also q-nbd of $\mathrm{x}_{\varepsilon}$ in $\tau_{i}$ and $v(\mathrm{r})+(\mu \vee \rho)(\mathrm{r})-1 \leq \ell_{1} \vee \ell_{2}(\mathrm{r})$, for every $\mathrm{r} \in \mathrm{X}$.

Therefore, by finite additively of fuzzy ideal as $\ell_{1} \vee \ell_{2} \in \mathrm{L}, \mathrm{x}_{\varepsilon} \notin(\mu \vee \rho)^{*}$. Hence $P(\mu \cup \rho)^{*}\left(\mathrm{~L}, \tau_{i}\right) \subseteq P \mu^{*}\left(\mathrm{~L}, \tau_{i}\right) \cup \rho^{*}\left(L, \tau_{i}\right)$. Clearly, both $\ldots$ and $\rho \subseteq \mu \cup \rho$ implies 
$P \mu^{*}\left(\mathrm{~L}, \tau_{i}\right) \cup \rho^{*}\left(L, \tau_{i}\right) \subseteq P(\mu \cup \rho)^{*}\left(\mathrm{~L}, \tau_{i}\right)$ and this completes the proof.

vi-Clear .

\section{Basic Structure Of Generated Fuzzy Bitopology}

Let $\left(\mathrm{X}, \tau_{1}, \tau_{2}\right)$ be a fbts and $\mathrm{L}$ be a fuzzy ideal on $\mathrm{X}$. Let us define $\tau_{i}-c l^{*}(\mu)=\mu \cup P \mu^{*}\left(L, \tau_{i}\right), i \in\{1,2\}$ for any fuzzy set $\mu \in I^{x}$. Clearly

$\tau_{i}-c l^{*}(\mu)$ is a fuzzy closure operator .Let $\tau^{*}{ }_{i}(L)$ be the fuzzy bitopology generated by $\tau_{i^{-}}$ $c l^{*}(\mu)$,i.e. $\tau^{*}{ }_{i}(L) \mid=\left\{\mu: \tau_{i}-c l^{*}\left(\mu^{c}\right)=\mu^{c}\right\}$. Now, $L=\left\{0_{x}\right\} \Rightarrow \tau_{i}$ $c l^{*}(\mu)=\mu \cup P \mu^{*}\left(L, \tau_{i}\right)=\mu \cup \tau_{i}-c l(\mu)=\tau_{i}-c l(\mu), i \in\{1,2\}$ for every $\mu \in I^{x}$, so $\tau^{*}{ }_{i}\left(\left\{0_{x}\right\}\right)=\tau_{i}, i \in\{1,2\}$.Again $L=I^{X} \Rightarrow$ $\tau_{i}-c l^{*}(\mu)=\mu \cup P \mu^{*}\left(L, \tau_{i}\right)=\mu \cup 0_{x}=\mu$, so $\tau_{i}^{*}\left(I^{X}\right), i \in\{1,2\}$ is fuzzy discrete bitopology on $X$.we can conclude by Theorem 2.1 (ii), $\tau^{*}{ }_{i}\left(\left\{0_{x}\right\}\right) \subseteq \tau^{*}{ }_{i}(L) \subseteq \tau^{*}{ }_{i}\left(I^{X}\right)$, i.e. $\tau_{i} \subseteq \tau_{i}{ }^{*}$, $L \subseteq J \Rightarrow \tau^{*}{ }_{i}(L) \subseteq \tau^{*}{ }_{i}(J)$.Let $\mu$ be a q-nbd of a fuzzy point $x_{\varepsilon}$ in $\tau^{*}{ }_{i}$ - fuzzy bitopology .Therefore, there exists $\rho \in \tau^{*}{ }_{i}, i \in\{1,2\}$ such that, and $\rho \subseteq \mu$, Now, $\mu \in \tau^{*}{ }_{i} \Leftrightarrow \mu^{c}$ is $\tau^{*}{ }_{i \text {-closed }} \Leftrightarrow \tau_{i}-c l^{*}\left(\mu^{c}\right)=\mu^{c} \Leftrightarrow P\left(\mu^{c}\right)^{*} \subseteq \mu^{c} \Leftrightarrow \mu \subseteq\left(P\left(\mu^{c}\right)^{*}\right)^{c}$.

Therefore $\varepsilon+\mu(x)>1 \Rightarrow \varepsilon+\left(\mu^{c}\right)^{*}(x)>1 \Rightarrow \varepsilon+1-P\left(\mu^{c}\right)^{*}(x)>1 \varepsilon>\left(\mu^{c}\right)^{*}(x) \Rightarrow$ $x_{\varepsilon} \notin\left(\mu^{c}\right)^{*}$. This implies there exists at least one q-nbd $v_{1}$ of $x_{\varepsilon}$ (in $\left.\tau_{\mathrm{i}}\right)$ such that for every $\mathrm{r} \in \mathrm{X}, v_{1}(\mathrm{r})$ $+\mu^{c}(\mathrm{r})-1 \leq \ell_{1}(\mathrm{x})$ for some $\ell_{1} \in$ L. i.e. $v_{1}(\mathrm{r})-\ell_{1}(\mathrm{r}) \leq \ell_{1}(\mathrm{x})$ for every $\mathrm{r} \in \mathrm{X}$ Therefore, as $v_{1} \mathrm{q}$-nbd of $x_{\varepsilon}$ (in $\tau_{\mathrm{i}}$ ), there is a $v \in \tau_{i}$ such that $x_{\varepsilon} q v \subseteq v_{1}$ and by heredity property of fuzzy ideal we have $\ell \in \mathrm{L}$ for which $x_{\varepsilon} q(v-\ell) \subseteq \mu$, where $(v-\ell)(r)=\max \{v(r)-\ell(r), 0\}$ for every $\mathrm{r} \in \mathrm{X}$. Hence, for $\mu \in \tau_{i}^{*}$, we have a $v \in \tau_{i}$ and $\ell \in \mathrm{L}$ such that,$(v-\ell) \subseteq \mu$. Let us denote $\beta\left(L, \tau_{i}\right)=\left\{v-\ell: v \in \tau_{i}, \ell \in L\right\}$.Then we have the following theorem

Theorem 3.1: $\beta\left(L, \tau_{i}\right)$ forms a basis for the generated fuzzy bitopology $\tau^{*}{ }_{i}(L)$ of the fbts $\left(\mathrm{X}, \tau_{1}, \tau_{2}\right)$ with fuzzy ideal $\mathrm{L}$ on $\mathrm{X}$, the class

$\beta\left(\mathrm{L}, \tau_{i}\right)=\left\{\mu-\lambda: \mu \in \tau_{i}, \lambda \in \mathrm{L}, i \in\{1,2\}\right\}$ is the base for the fuzzy bitopology $\tau_{i}{ }^{*}$

Proof: Straight forward

Theorem 3.2. If $\mathrm{L}_{1}$ and $\mathrm{L}_{2}$ are two fuzzy ideals on fbt $\left(\mathrm{X}, \tau_{1}, \tau_{2}\right)$ then,

i- $\quad \mathrm{P} \mu^{*}\left(\mathrm{~L}_{1}, \tau_{i}\right) \geq \mathrm{P} \mu^{*}\left(\mathrm{~L}_{2}, \tau_{i}\right)$ for every $\mu \in \mathrm{I}^{\mathrm{X}}$ and $\mathrm{L}_{1} \leq \mathrm{L}_{2}$.

ii- $\quad\left(\mathrm{L}_{1}\right) \leq \tau i^{*}\left(\mathrm{~L}_{2}\right)$ and $\mathrm{L}_{1} \leq \mathrm{L}_{2}$.

iii- $\quad P \mu^{*}\left(L_{1} \cap L_{2}, \tau_{i}\right)=P \mu^{*}\left(L_{1}, \tau_{i}\right) \cup P \mu^{*}\left(L_{2}, \tau_{i}\right)$.

iv- $P \mu^{*}\left(L_{1} \vee L_{2}, \tau_{i}\right)=P \mu^{*}\left(L_{1}, \tau^{*}{ }_{i}\left(L_{2}\right)\right) \cap P \mu^{*}\left(L_{2}, \tau^{*}{ }_{i}\left(L_{1}\right)\right)$.

Proof. i and ii are clear.

iii-Let $\mathrm{x}_{\varepsilon} \notin, P \mu^{*}\left(\mathrm{~L}_{1}, \tau_{i}\right) \cup P \mu^{*}\left(L_{2}, \tau_{i}\right)$. So $\mathrm{x}_{\varepsilon}$ is not contained in both $P \mu^{*}\left(\mathrm{~L}_{1}, \tau_{i}\right)$ and $P \mu^{*}\left(L_{2}, \tau_{i}\right)$.

Now $\mathrm{x}_{\varepsilon} \notin P \mu^{*}\left(\mathrm{~L}_{1}, \tau_{i}\right)$ implies there is at least one $\mathrm{q}-\mathrm{nbd} v_{1}$ in $\tau_{i}$, of $\mathrm{x}_{\varepsilon}$ such that for every $\mathrm{r} \in \mathrm{X}, v_{1}(\mathrm{r})$ 
$+\mu(\mathrm{r})-1 \leq \ell_{1}(\mathrm{r})$, for some $e_{1} \in \mathrm{L}$. Again $\mathrm{x}_{\varepsilon \notin \mathrm{P \mu}^{*}}\left(\mathrm{~L}_{2}, \tau_{i}\right)$ and similarly, there is at least one q-nbd $v_{2}$ of $\mathrm{x}_{\varepsilon}$ in $\tau_{i}$ such that, for every $\mathrm{r} \in \mathrm{X}, v_{2}(\mathrm{r})+\mu(\mathrm{r})-1 \leq \ell_{2}(\mathrm{x})$ for some $\ell_{2} \in \mathrm{L}$. Therefore, we have $v=v_{1} \cap v_{2}$, so $v$ is also q-nbd of $\mathrm{x}_{\varepsilon}$ in $\tau_{i}$ and $v(\mathrm{r})+\mu(\mathrm{r})-1 \leq \ell_{1} \cap \ell_{2}(r)$ for every $\mathrm{r} \in \mathrm{X}$. Since $v$ is also q-nbd of $\mathrm{x}_{\varepsilon}$ in $\tau_{i}$ and $\ell_{1} \cap \ell_{2} \in v$, therefore $\mathrm{x}_{\varepsilon} \notin P \mu^{*}\left(L_{1} \cap L_{2}, \tau_{i}\right)$,so that $P \mu^{*}\left(L_{1} \cap L_{2}, \tau_{i}\right) \subseteq P \mu^{*}\left(L_{1}, \tau_{i}\right) \cup P \mu^{*}\left(L_{2}, \tau_{i}\right)$. Also $L_{1} \cap L_{2}$ included in both $L_{1}$ and $L_{2}$, so by Theorem 2.2(ii), reverse inclusion is obvious, which completes the proof of (iii)

$\mathrm{v}$ - $\quad$ iv) Let $\mathrm{x}_{\varepsilon} \notin, P \mu^{*}\left(\mathrm{~L}_{1} \vee L_{2}, \tau_{i}\right)$ implies there is at least one q-nbd $v_{1}$ of $\mathrm{x}_{\varepsilon}$ in $\tau_{i}$ such that for every $\mathrm{r} \in \mathrm{X}, v_{1}(\mathrm{r})+\mu(\mathrm{r})-1 \leq \ell_{1}(\mathrm{r})$, for some $\ell_{1} \in \mathrm{L}_{1} \vee L_{2}$. Therefore, by heredity of fuzzy ideals and considering the structure of fuzzy $\tau_{i-}$ open sets generated fuzzy bitopology, we can find $v_{1}, v_{2}$ the q-nbd of $\mathrm{x}_{\varepsilon}$ in $\tau^{*}{ }_{i}\left(L_{1}\right)$ or $\tau^{*}{ }_{i}\left(L_{2}\right)$ respectively, such that, for every $\mathrm{r} \in \mathrm{X}, v_{1}(\mathrm{r})+\mu(\mathrm{r})-1 \leq \ell_{1}(\mathrm{r})$ or $v_{2}(\mathrm{r})+\mu(\mathrm{r})-1 \leq \ell_{2}(\mathrm{r})$ for some $\ell_{2} \in \mathrm{L}_{2}$ or $\ell_{1} \in \mathrm{L}_{1}$ for every $\mathrm{r} \in \mathrm{X}$. This implies

$\mathrm{x}_{\varepsilon} \notin P \mu^{*}\left(L_{1}, \tau^{*}{ }_{i}\left(L_{2}\right)\right)$ or $\mathrm{x}_{\varepsilon} \notin P \mu^{*}\left(L_{2}, \tau^{*}{ }_{i}\left(L_{1}\right)\right)$. Thus we have, $P \mu^{*}\left(L_{1}, \tau^{*}{ }_{i}\left(L_{2}\right)\right) \cap P \mu^{*}\left(L_{2}, \tau^{*}{ }_{i}\left(L_{1}\right)\right) \subseteq P \mu^{*}\left(L_{1} \vee L_{2}, \tau_{i}\right)$.

Conversely, let $\mathrm{x}_{\varepsilon} \notin P \mu^{*}\left(L_{1}, \tau^{*}{ }_{i}\left(L_{2}\right)\right)$. This implies there is least one on q-nbd $v$ of $\mathrm{x}_{\varepsilon}$ in $\tau^{*}{ }_{i}$ such that for every $\mathrm{r} \in \mathrm{X}, v(\mathrm{r})+\mu(\mathrm{r})-1 \leq \ell_{1}(\mathrm{r})$, for some $\ell_{1} \in \mathrm{L}_{1}$. Since $v$ is a $\tau^{*}{ }_{i}\left(L_{2}\right) \mathrm{q}$-nbd of $\mathrm{x}_{\varepsilon}$, by heredity of fuzzy ideals, we have a q-nbd $v^{\prime}$ of $\mathrm{x}_{\varepsilon}$ in $\tau_{i}$ such that for every $\mathrm{r} \in \mathrm{X}, v(\mathrm{r})+\mu(\mathrm{r})-1 \leq \ell_{1} \cup \ell_{2}(\mathrm{r})$, for some $\ell_{1} \in \mathrm{L}_{1}$ and for some $\ell_{2} \in \mathrm{L}_{2}$.i.e., $\mathrm{x}_{\varepsilon} \notin, P \mu^{*}\left(\mathrm{~L}_{1} \vee L_{2}, \tau_{i}\right)$.Thus, $P \mu^{*}\left(\mathrm{~L}_{1} \vee L_{2}, \tau_{i}\right) \subseteq P \mu^{*}\left(L_{1}, \tau^{*}{ }_{i}\left(L_{2}\right)\right)$ and $P \mu^{*}\left(L_{2}, \tau^{*}{ }_{i}\left(L_{1}\right)\right)$.Then $P \mu^{*}\left(\mathrm{~L}_{1} \vee L_{2}, \tau_{i}\right) \subseteq P \mu^{*}\left(L_{1}, \tau^{*}{ }_{i}\left(L_{2}\right)\right) \cap P \mu^{*}\left(L_{2}, \tau^{*}{ }_{i}\left(L_{1}\right)\right)$ and this completes the proof .

An important result follows form the above theorem that $\tau_{i}{ }^{*}(L)$ and $\tau_{i}^{* *}(L)$ are

Equal for any fuzzy ideal on X.

Corollary 3.1: Let $\left(\mathrm{X}, \tau_{1}, \tau_{2}\right)$ be a fbts with fuzzy ideal L. Then $\tau_{i}^{*}(L)=\tau_{i}^{* *}(L)$

Proof. By taking $\mathrm{L}_{1}=\mathrm{L}_{2}=\mathrm{L}$ in the above Theorem, we have the required result

Corollary 3.2. . If $\mathrm{L}_{1}$ and $\mathrm{L}_{2}$ are two fuzzy ideals on fbt $\left(\mathrm{X}, \tau_{1}, \tau_{2}\right)$ then,

i- $\quad \tau_{i}^{*}\left(L_{1} \vee L_{2}, \tau_{i}\right)=\left[\tau_{i}^{* *}\left(L_{2}, \tau_{i}\right)\right]\left(L_{1}\right)=\left\lfloor\tau_{i}^{* *}\left(L_{1}, \tau_{i}\right)\right\rfloor\left(L_{2}\right)$,

ii- $\quad \tau_{i}^{*}\left(L_{1} \vee L_{2}, \tau_{i}\right)=\left[\tau_{i}^{*}\left(L_{1}, \tau_{i}\right)\right] \vee\left[\tau_{i}^{*}\left(L_{2}, \tau_{i}\right)\right]$,

iii- $\quad \tau_{i}^{*}\left(L_{1} \cap L_{2}, \tau_{i}\right)=\left[\tau_{i}^{*}\left(L_{1}, \tau_{i}\right)\right] \cap\left[\tau_{i}^{*}\left(L_{2}, \tau_{i}\right)\right]$

\section{Proof .Clear}

\section{References}

[1] M. K. Chakraborty and T.M.G. Ahasanullah, Fuzzy topology on fuzzy sets and tolerance topology, Fuzzy Sets and Systems 45 (1992) 103-108.

[2] C. L. Chang, Fuzzy Topological Spaces, J. Math. Anal. Appl. 24 (1968) 128 - 189.

[3] Pu Pao - Ming and Liu Ying, Fuzzy Topology.1. Neighbourhood structure of a fuzzy point and moore smith convergence, J Math. Anal. Appl. 76 (1980) 571 - 599.

[4] A. A. Nouh, On separation axioms in fuzzy bitopological spaces, Fuzzy Sets and Systems, 80(1996)225-236.

[5] Debasis Sarkar, Fuzzy ideal theory, Fuzzy local function and generated fuzzy topology, Fuzzy Sets and Systems 87 (1997) 117 $-123$.

[6] L. A. Zadeh, Fuzzy Sets Inform. and Control 8 (1965) 338 - 353. 